www.eeplt.edu.pe/revista/

\title{
Factores de riesgo de violencia de género en adolescentes de educación secundaria, Manchay
}

\author{
María Inés Ruiz Garay, ${ }^{\mathrm{a}, \mathrm{b}}$ Esther Cecilia García Mercado, ${ }^{\mathrm{a}, \mathrm{b}}$ Yojana Ruiz Merino, ${ }^{\mathrm{c}, \mathrm{b}}$ Guillermina Valdivia Arce, ${ }^{\mathrm{c}, \mathrm{b}}$ \\ Liliana Rina Tippe Quispe, ${ }^{\mathrm{c} b}$ Lizbeth Córdova-Berrú, ${ }^{\mathrm{c}, \mathrm{a}}$ Clery Liz Palacios Saavedra. ${ }^{\mathrm{c}}$
}

\begin{abstract}
Ruiz GMI, García MEC, Ruíz MY, Valdivia AG, Tippe QLR, Córdova-Berrú $L$ et al. Factores de riesgo de violencia de género en adolescentes de educación secundaria, Manchay. Cuid salud, ene-jun 2015; 2(1):156-163.
\end{abstract}

\section{RESUMEN}

La violencia constituye un problema de salud pública, que afecta más a las mujeres con grave impacto en su esfera física-psico-emocional. La OPS identifica factores de riesgo de violencia en diversos contextos, desde las características individuales, pasando por el seno familiar, hasta las políticas del país. Objetivo: determinar la presencia de factores de riesgo de violencia de género en adolescentes de educación secundaria, Manchay. Metodología: estudio de enfoque cuantitativo, diseño exploratorio descriptivo; cuya población fue de 267 adolescentes mujeres de tres colegios de la UGEL $\mathrm{N}^{\circ} 1$. Para recolectar los datos se utilizó la encuesta y un cuestionario con tres factores reconocidos: individuales (14), relacionales (21) y sociales (10); con respuestas ordinales con puntaje máximo 230 y mínimo 45 . Los datos se recolectaron entre octubre-diciembre 2013, siendo analizados mediante estadística descriptiva en paquete SPSS versión 17.0. El proyecto fue aprobado por un comité de ética universitario. Resultados: factor individual: el $70,8 \%$ de adolescentes estaba insatisfecha con su imagen corporal y $52 \%$ con su rendimiento escolar, 73,4\% consideraba más fácil dialogar con su padre y $63 \%$ no cumplía con las tareas encomendadas. Relacional: el 81,8\% refirió que en casa se impone quien más grita, $67,7 \%$ consideró las actitudes de amigos inadecuadas. Social: el $58,5 \%$ centraba su atención en noticias policiales, $48 \%$ consideraba que las leyes no siempre protegen a la mujer. Conclusiones: los factores de riesgo individual y social están presenten con mayor proporción en las adolescentes. Es necesario intervenir en el seno familiar para prevenir la violencia de género.
Ruiz GMI, García MEC, Ruíz MY, Valdivia AG, Tippe QLR, Córdova-Berrú $L$ et al. Risk factors of gender violence in adolescents of high school. Manchay. Cuid salud, ene-jun 2015; 2(1):156-163.

\begin{abstract}
Violence is a public health problem that affects more women with serious emotional impact on their psycho-physical areas. PAHO identifies risk factors for violence in various contexts, from the individual characteristics, to the family, to the country's policies. Objective: To determine the presence of risk factors of gender violence in adolescent of high school, Manchay. Methodology: study of quantitative approach, descriptive exploratory design; whose population was 267 adolescent girls from three schools in the UGELs No. 1. To collect data survey and a questionnaire with three recognized factors used: individual (14), relational (21) and social (10); with ordinal responses with maximum score (230) and minimum (45). Data were collected between October to December / 2013, and were analyzed using descriptive statistics in SPSS version 17.0 package. The project was approved by a university ethics committee. Results: Individual factor: $70.8 \%$ of teenagers were dissatisfied with their body image and 52\% with their school performance, $73.4 \%$ considered easier to talk with his father and $63 \%$ did not comply with the tasks. Relational: $81.8 \%$ reported that prevails at home who shouts the most, $67.7 \%$ considered inadequate attitudes of friends. Social: $58.5 \%$ focused attention on police news, $48 \%$ thought that laws do not always protect women. Conclusions: individual and social factors of risk are present with a higher proportion in adolescents. It is necessary to intervene in the family to prevent gender violence.
\end{abstract}

Keywords: Violence, gender violence, adolescence and prevention.

Palabras clave: violencia, violencia de género, adolescencia, prevención.

\footnotetext{
${ }^{a}$ Magíster en Enfermería.

${ }^{\text {b }}$ Docente de la Escuela de Enfermería Padre Luis Tezza afiliada a la Universidad Ricardo Palma, Lima-Perú.

${ }^{\mathrm{c}}$ Licenciada Enfermera.

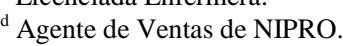

${ }^{\mathrm{e}}$ Emergencista de Sistema de Atención Móvil de Urgencias (SAMU).
} 


\section{INTRODUCCIÓN}

Las Naciones Unidas refieren que la violencia contra la mujer comprende todo acto de violencia basado en la pertenencia al sexo femenino, que está vinculado al poder, privilegio y control masculino; afecta a las mujeres independiente de su edad, color, raza, religión, nacionalidad, opción sexual o condición social, ocasionando como resultado un daño o sufrimiento físico, sexual o psicológico; así como las amenazas de tales actos, la coacción o la privación arbitraria de la libertad, tanto en la vida pública como en la privada. ${ }^{1}$

Dicha violencia es denominada violencia de género y está presente en casi todos los países, con una prevalencia mundial de $35 \%$ y puede ser de carácter físico, sexual y psicológico, ${ }^{2}$ con mayor número de experiencias violentas al interior de los países y entre ellos se encuentra el Perú. Siendo que las mujeres jóvenes de 15-19 años tienen mayor riesgo de sufrir violencia física/sexual -o ambas- perpetrado por la pareja, hecho que ocurre con mayor frecuencia en el medio urbano del Perú. ${ }^{3}$ Datos recientes del Programa Nacional contra la Violencia Familiar y Sexual muestran que 1423 niños, niñas y adolescentes fueron atendidos por causa de violencia, con mayor incidencia de la psicológica, seguida de la sexual y física. ${ }^{4}$

Con frecuencia la violencia psicológica es precedida de la violencia física, ${ }^{5}$ la misma que puede terminar en algunos casos en femenicidio. Walker, citada por Alonso, ${ }^{6}$ identifica que en la violencia existe un proceso de interacción mutua entre el agresor y la víctima, produciéndose lo que se denomina "síndrome de la mujer maltratada", caracterizada por un aumento de la respuesta fisiológica, aislamiento, fatalismo, reducción progresiva de la autoestima e indefensión aprendida; dichos cambios en la esfera psicológica constituyen uno de los factores responsables de que la mujer continúe en la relación de abuso/maltrato a lo largo de su vida.

Núñez ${ }^{7}$ refiere que en el origen del maltrato a la mujer pueden considerarse tres elementos: el agresor, la víctima y las circunstancias del contexto. En este último se encuentran los condicionantes socioculturales que actúan sobre el género masculino y femenino, situando a la mujer en una posición de subordinación, que legitima la violencia al considerar al varón como superior por naturaleza y quien debe dirigir la conducta de la mujer. $^{8}$

Estereotipos y mitos culturales que se perpetúan en la sociedad a través de las instituciones, representadas por una serie de espacios en cuya estructura y funcionamiento se internalizan comportamientos que reproducen el modelo de poder vertical y autoritario al resolver sus conflictos institucionales. Aquí se pueden citar las escuelas, espacios recreativos, laborales, religiosos, judiciales y los medios de comunicación, como el internet, la televisión y los carteles publicitarios; los cuales tienen una influencia decisiva en la generación de actitudes y en la legitimación de conductas violentas en la sociedad, especialmente entre los niños y adolescentes, quienes son testigos y reproductores de modelos relacionales, sobre todo ante la carencia de una red familiar y social de apoyo.

Corsi, citado por Nóblega y Núñez, ${ }^{9}$ señala que la verticalidad de la familia, la rigidez en sus relaciones, la adhesión a roles de género tradicionales, las creencias en torno a la obediencia y el respeto, la disciplina y el valor del castigo, así como la autonomía de los miembros, son elementos que predisponen a la familia a relaciones violentas.

Estudios de revisión sobre violencia en jóvenes revelan que las víctimas de violencia o haber sido testigo de violencia entre los padres, en la familia de origen o fuera de ella (relaciones con los pares), favorece la realización de actos violentos por parte de los adolescentes y adultos jóvenes. ${ }^{5} \mathrm{Al}$ respecto, Tenorio ${ }^{4}$ resalta que para reducir el impacto de la violencia contra la mujer es necesario intervenir en la etapa adolescente, dado que comienzan a desvincularse de la familia y priorizan sus relaciones con los pares; etapa en que también comienzan los enamoramientos y, sin percatarse se vinculan con varones que tienen comportamientos violentos disfrazados de "normales" en la actual sociedad, como los celos, agresiones verbales, manipulaciones sentimentales y desapego de sus amistades (posesión); todas estas situaciones enmascaran actos de violencia.

Frente a esto, la prevención de la violencia de género es un asunto que compete a todos, a la familia y la sociedad en general; pues constituye un problema de salud pública por las graves repercusiones que ocasiona en las víctimas y sus hijos, extendiéndose hacia la sociedad. ${ }^{1}$ Con impacto económico en el país, dado que las víctimas son atendidas en el sistema de salud por patologías que llegan a somatizar por causa del sufrimiento que experimentan, entre ellas la depresión; otro aspecto es la productividad en el ambiente laboral y tal vez su mayor repercusión es generada en el seno familiar, afectando en igual medida a la socialización y aprendizaje de los 
niños, quienes pueden desarrollar baja autoestima e incapacidad para confiar y amar a otros. ${ }^{11} \mathrm{La}$ ENDES-2014 ${ }^{2}$ reportó que el $30,7 \%$ de madres con hijos/as menores de cinco años ha sufrido violencia física y/o sexual y, que el 38,6\% de ellas había declarado que su menor fue testigo de la agresión.

Si bien, el Estado ha implementado el Plan Nacional Contra la Violencia hacia la Mujer, se debe poner énfasis en el aspecto de la prevención, ya que los adolescentes constituyen un grupo mayoritario y muchas veces no existen políticas claras que lo protejan en esta etapa, que es la más fascinante y compleja de la vida, en la cual se busca la identidad y se práctica los valores aprendidos en la infancia. Es en esta vorágine de cambios que debe enfrentarse al contexto sociocultural, de género, situación económica, entre otros. $^{12}$

Razón imperante para actuar en el hogar e identificar los factores que constituyen riesgo latente para las adolescentes, futuras madres de familia y profesionales que contribuirán en el cambio del tejido social, basado en el respeto, aceptación y disminución de las inequidades sociales. El objetivo de este estudio fue identificar la presencia de factores de riesgo para la violencia de género en adolescentes de educación secundaria, en la comunidad de Manchay, considerando el precedente que el $77,5 \%$ de hombres de esta comunidad presentaba una actitud favorable hacia la violencia, la cual era justificada por el dominio patriarcal heredado de sus ancestros. $^{13}$

Para el análisis de los factores se tomó como referente el modelo ecológico de la violencia propuesto por la Organización Panamericana de la Salud (OPS), ${ }^{14}$ que concibe a la violencia desde una perspectiva polifacética integral, en la que interaccionan múltiples factores como las individuales y relacionales, que influyen y son influenciados por la comunidad y la sociedad. En el primer nivel del modelo (más interna), se identifican los factores biológicos y la historia personal del sujeto, como las características sociodemográficas, trastornos psíquicos $\mathrm{o}$ de personalidad, toxicomanías y antecedentes de haber sido agredido/agresivo; en el segundo nivel están las relaciones más próximas del sujeto con su familia, amigos, compañeros y pareja; en el tercer nivel se exploran los contextos comunitarios, identificándose aquí al vecindario, la escuela, el espacio laboral, entre otros $y$, finalmente, en el cuarto nivel se consideran las estructuras de la sociedad que inhiben o alientan la violencia, entre ellos las normas sociales y culturales, las políticas sanitarias, económicas y educativas. Existiendo interconexiones entre los niveles que aumentan o reducen el riesgo de violencia.

\section{METODOLOGÍA}

Estudio de enfoque cuantitativo y método observacional, de diseño exploratorio descriptivo; realizado en tres colegios mixtos estatales de nivel primario y secundario, localizados en Manchay, distrito de Pachacámac, Provincia de Lima-Perú. La población fue constituida por estudiantes mujeres de secundaria $(\mathrm{N}=800)$, siendo los criterios de inclusión: estar cursando entre 3ro y 5to año de secundaria, con asistencia regular a clase, turno diurno, edad entre 14-17 años, participación voluntaria y contar con la autorización de sus padres. Como exclusión se consideró residencia en el área menor de seis meses. De este modo, la muestra fue censal constituida por 267 estudiantes.

La técnica empleada para recolectar los datos fue la encuesta y el instrumento un cuestionario elaborado por las autoras basado en la revisión de la literatura, el cual fue sometido a juicio de expertos (10 profesionales de la salud), cuyos resultados se analizaron con la prueba binomial, existiendo concordancia en la mayoría de los criterios de evaluación entre los expertos. Posteriormente, se desarrolló confiabilidad del instrumento mediante estudio piloto con 30 adolescentes que no formaron parte del estudio final, resultados que fueron analizados con el coeficiente Alpha de Cronbach $(p=0,79)$. El instrumento quedó constituido de 49 ítems: 04 correspondientes a datos generales y 45 a los factores de riesgo de violencia: individual (14), relacional (21) y social (10). Cada respuesta se calificaba en una escala de graduación (1-5 puntos), obteniéndose como puntaje máximo 230 y mínimo 45 .

Para aplicar la encuesta se cumplieron con los procedimientos administrativos en cada institución educativa, siendo los datos recolectados por una de las investigadoras durante los meses de octubrediciembre del 2013, previa coordinación con los docentes y tutores para la obtención del Consentimiento Informado con los padres de familia $y$ el Asentimiento Informado con los adolescentes. El llenado del cuestionario con cada grado de estudio tomó un tiempo aproximado de 30 minutos; destacando que cualquier duda con los adolescentes respecto al estudio fue absuelta antes del llenado. 
Previo al análisis de datos, se realizó el control de calidad verificando la información obtenida; cada cuestionario fue codificado al igual que los datos con la finalidad de preservar el anonimato de los participantes; luego se digitaron en una base de datos en hoja de cálculo Excel 2010. Finalmente, se transportaron al programa SPSS versión 17.0 y procesados mediante la estadística descriptiva (porcentaje, frecuencia, media, desviación estandar). Dado la polarización de los datos diseñados para una escala, estas se dicotomizaron para el análisis final.

El estudio fue aprobado por el Comité de Ética de la Facultad de Medicina de la Universidad Ricardo Palma (Oficio N N 01142013/CE), cumpliéndose con los principios éticos aplicados a la investigación: Beneficencia porque se buscó conocer los factores que podrían predisponerla a ser víctima de violencia a las adolescentes, a fin de proponer intervenciones futuras; Autonomía porque no sufrieron coacción para participar en el estudio; Justicia porque todas las adolescentes tuvieron la oportunidad de participar y fueron tratadas con igualdad sin discriminación; No maleficencia porque no sufrieron daño físico y/o mental, el estudio contó con una psicóloga para apoyar en caso que la adolescente lo requiriera.

\section{RESULTADOS}

La edad media de las adolescentes fue de $15 \pm 1,18$ años de desvío padrón (DP), el número de ambientes por casa fue de 7,02 $\pm 1,89 \mathrm{DP}$, considerando un rango de hermanos entre 3-5, con una moda de 4 hijos por familia.

Tabla 1: Datos generales de las adolescentes de educación secundaria de Manchay. OctubreDiciembre 2013.

\begin{tabular}{|c|c|c|c|}
\hline & Características & $\begin{array}{c}\text { Frecuencia } \\
(n=267)\end{array}$ & $\begin{array}{c}\text { Porcentaje } \\
(100 \%)\end{array}$ \\
\hline \multirow{5}{*}{ 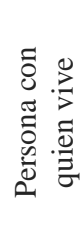 } & Papá, mamá, hermanos & 189 & 70,8 \\
\hline & Solo con mamá & 47 & 17,6 \\
\hline & Solo con papá & 5 & 1,9 \\
\hline & Con madrastra, padrastro & 5 & 1,9 \\
\hline & Otros & 18 & 6,7 \\
\hline \multirow{4}{*}{ 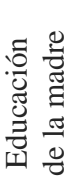 } & Superior & 21 & 7,9 \\
\hline & Secundaria & 109 & 40,8 \\
\hline & Primaria & 107 & 40,1 \\
\hline & Iletrada & 5 & 1,9 \\
\hline \multirow{4}{*}{ 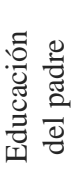 } & Superior & 40 & 15,0 \\
\hline & Secundaria & 130 & 48,7 \\
\hline & Primaria & 66 & 24,7 \\
\hline & Iletrado & - & - \\
\hline
\end{tabular}

En la tabla 1, se identifica que más del $70 \%$ de adolescentes vivía con ambos padres y hermanos; el 40,8\% de madres tenía estudios secundarios y en los padres este nivel alcanzó el 48,7\%.

Gráfico 1. Factores de riesgo de violencia de género en las adolescentes de secundaria. Manchay, octubre - diciembre 2013.

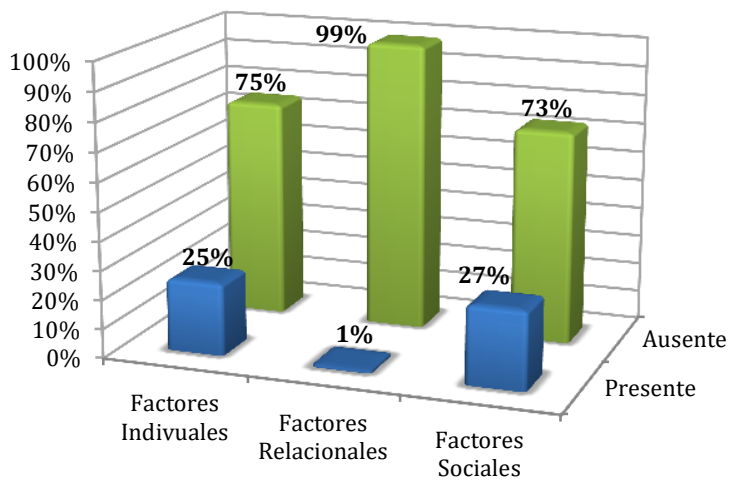

En el gráfico 1, tanto el factor "social" como el "individual" se encuentran presentes como riesgos potenciales de violencia de género en el $27 \%$ (72) y 25\% (67) de las adolescentes, respectivamente.

Gráfico 2. Factor de riesgo individual en las adolescentes de secundaria. Manchay, octubre diciembre 2013.
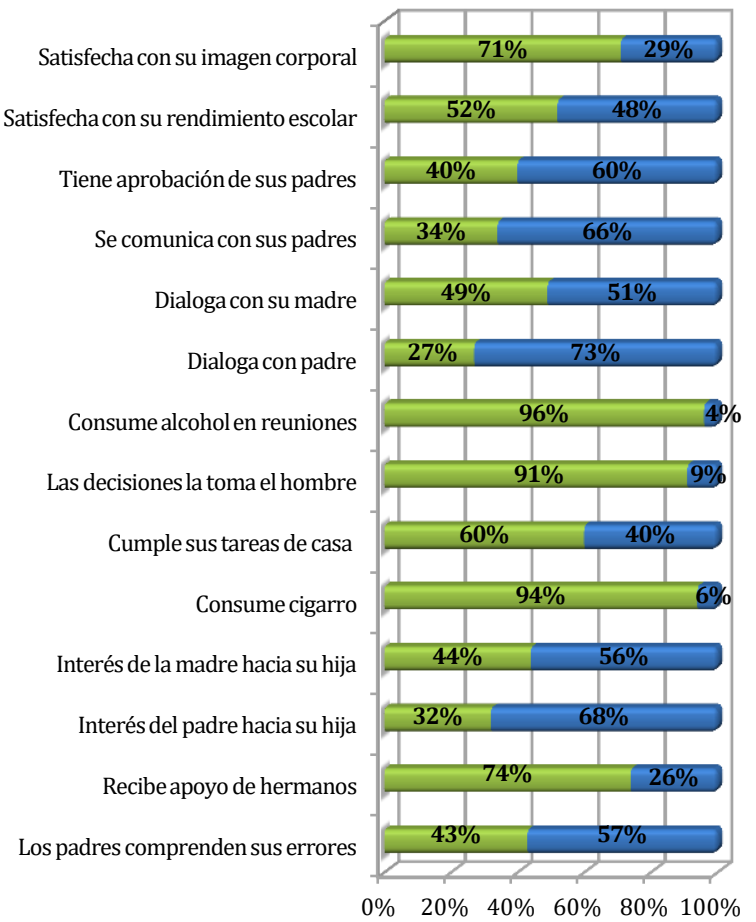

$\square$ No $\square \mathrm{Si}$ 
En el gráfico 2, las adolescentes no estaban satisfechas con su imagen corporal y su rendimiento escolar. Asimismo, dialogan más con el padre que con la madre ante las dificultades de la vida, un importante porcentaje no realiza las tareas de casa, en general los padres muestran interés por las cosas de sus hijas, pero un $44 \%$ (117) de madres hace descaso de este aspecto, dato semejante se evidencia respecto a la comprensión de los errores de la joven.

Gráfico 3. Factor de riesgo social en las adolescentes de secundaria. Manchay, octubre diciembre 2013.

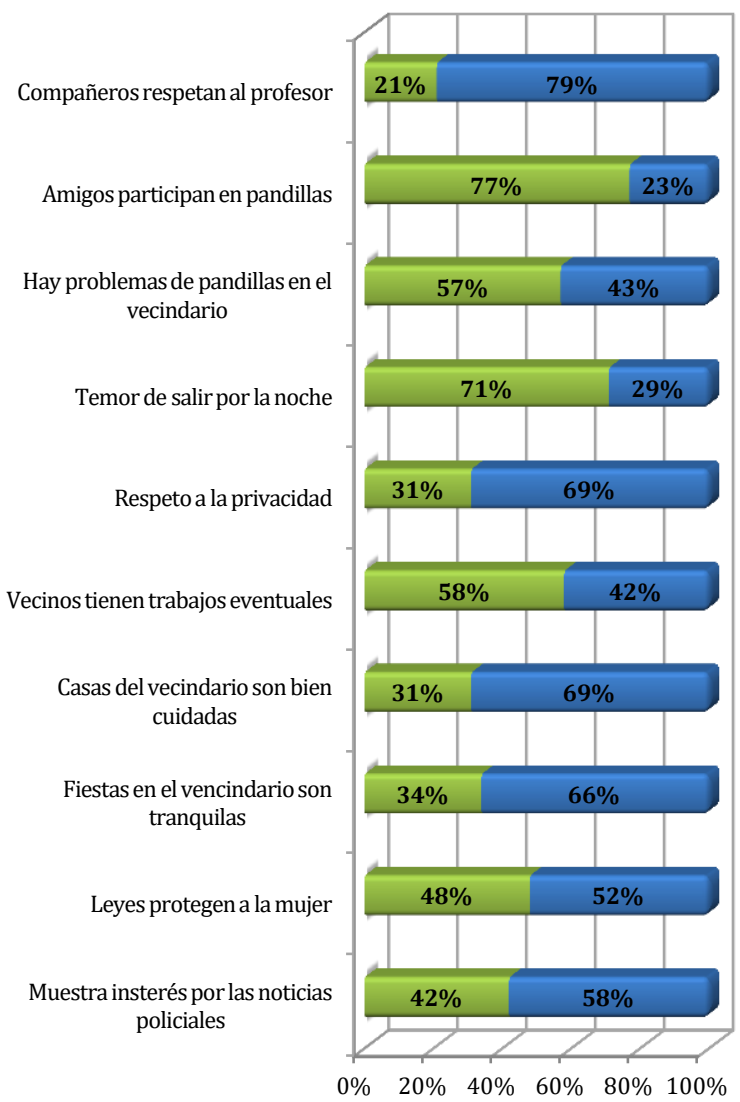

$\square$ No $\square \mathrm{Si}$

En el gráfico 3, se resalta que en la escuela aproximadamente el 30\% no respeta al profesor, siendo que un importante porcentaje $(23 \% / 61)$ de amigos participa en pandillas, situación que se ve también en el vecindario, aunque por otro lado, las adolescentes refieren que se respeta la privacidad. En general, las casas son bien cuidadas y las fiestas ocurren de manera tranquila, pero un $34 \%$ (90) dijo lo contrario; un importante porcentaje considera que las leyes no protegen a la mujer y que suelen estar atentas a las noticias policiales que se difunden en los medios de comunicación.
Gráfico 4. Factor de riesgo relacional en las adolescentes de secundaria. Manchay, octubre diciembre 2013.

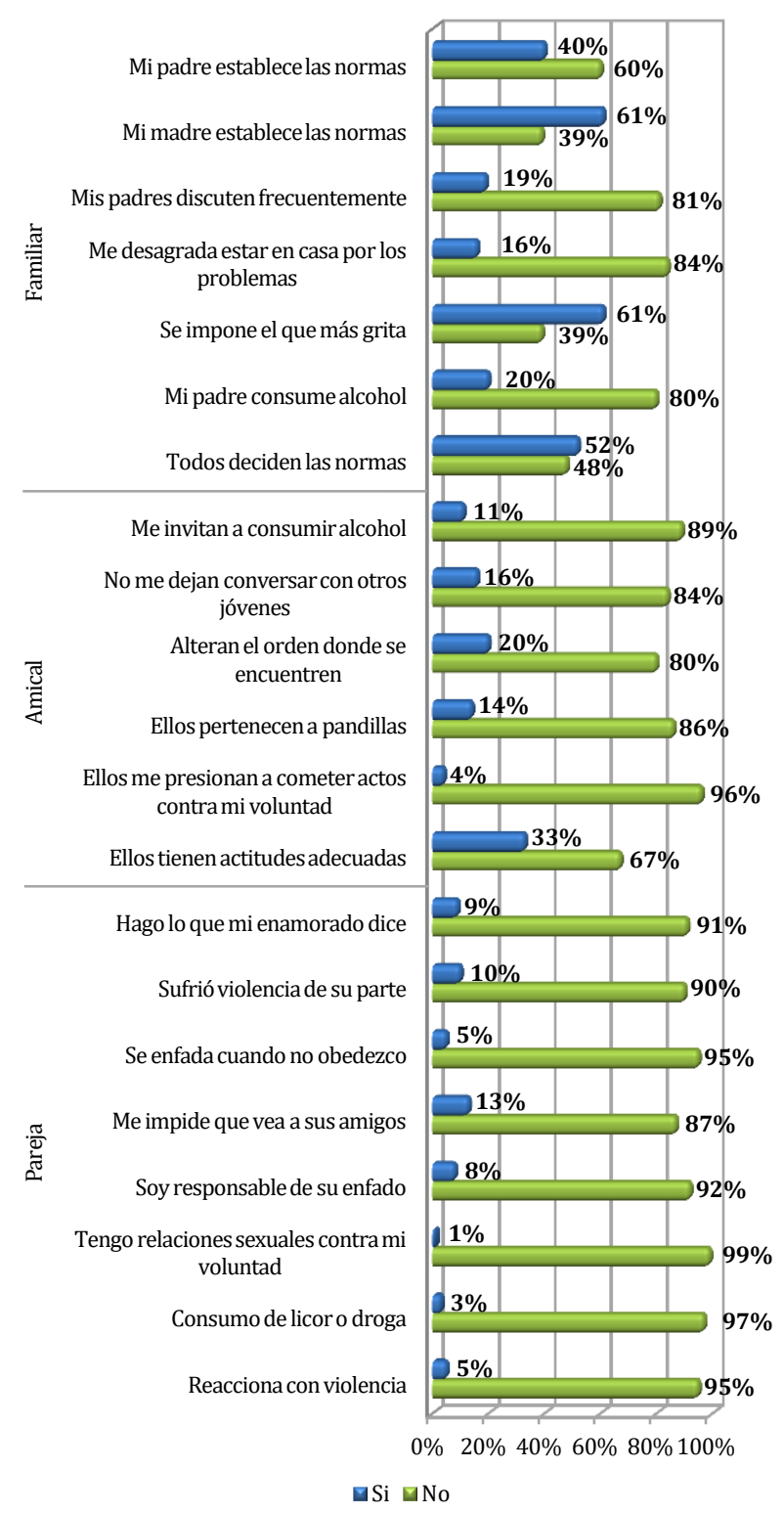

En el gráfico 4, en la dimensión familiar las adolescentes refieren que la madre establece las normas en el hogar y un significativo porcentaje $(19 \% / 51)$ refirió que sus padres discuten con frecuencia y que, en general, en casa se impone el que más grita, frente al hecho de que "todos deciden las normas en casa". Se identifica también que hay presencia de consumo de alcohol de parte del padre. En la dimensión amical, las adolescentes consideran que las actitudes de sus amigos son inadecuadas, siendo que más del $10 \%$ de ellos pertenecen a pandillas, otros $20 \%$ alteran el orden en donde se encuentran. Respecto a la dimensión pareja, aproximadamente el $10 \%$ de adolescentes tiene problemas de violencia con su pareja, como se ve en los datos ("Hago lo que mi enamorado 
dice", "Sufrió violencia de su parte", "Me impide que vea a sus amigos" y "Soy responsable de su enfado") y el $5,1 \%$ reportó que reacciona con violencia ante los problemas y/o dificultades del día a día.

\section{DISCUSIÓN}

Manchay es una comunidad conformada por una población migrante venida de la sierra sur y central del país, quienes huían de la pobreza y la violencia interna ocurrida en el Perú en la década del 80; población que mantiene sus raices culturales matizadas con el sufrimiento que generó el éxodo, formas que a veces no son comprendidas en la modernidad del mundo de hoy. ${ }^{15}$ Actualmente, aún existen zonas carentes de saneamiento básico, viviendas de material precario y las personas suelen tener prácticas costumbristas del lugar de origen; observándose predominio del consumo excesivo de alcohol.

Queda claro que la violencia es una conducta social aprendida y que ésta a su vez influye en la construcción de la personalidad individual, reconociéndose que en dicho proceso intervienen patrones comportamentales como la endoculturación y la educación; con gran dominio de la cultura intergeneracional. ${ }^{16,} 17 \quad$ Esta construcción suele iniciarse en la adolescencia y termina consolidándose en la tercera edad, ${ }^{17}$ lo que justamente preocupa y convoca la realización del estudio.

Los resultados muestran que el factor individual (gráfico 2) y social (gráfico 3) sobresalen frente al relacional. Existe una insatisfacción de la adolescente consigo misma, con escaso soporte de los padres en sus asuntos personales y desinterés de la madre. La actual influencia mediática de la imagen corporal que venera la belleza nórdica en desmedro de la peruana puede estar presente en este rechazo, así como el desvinculamiento de la familia en el desarrollo de sus funciones con la hija; Chávez Asencio, citado por Salas, ${ }^{18}$ refiere que el objetivo de la familia es formar personas, educar con fe y principios para así lograr el desarrollo integral de los hijos, proceso que se determina en la socialización de la escuela primaria y se afianza en la secundaria; cuanto más estrecha sea la relación del adolescente con sus padres, tendrán mayor iniciativa social y autoestima positiva, 12 sintiéndose más seguros de sí mismos, lo que se constituye en factor protector porque evita su identificación con pares que podrían poner en riesgo la integridad de las adolescentes.
Llama la atención en el estudio las respuestas contradictorias que registraron las adolescentes. Por un lado, la presencia de pandillas en la escuela y en el vecindario (gráfico 3), los cuáles en ocasiones llegan a alterar el orden (gráfico 4). Por otro, algunas de ellas observan que el vecindario es tranquilo y seguro por las noches, pero prestan gran atención a las noticias policiales (gráfico 3), siendo que mantienen amistades con jóvenes pandilleros y reconocen que la mayoría de amigos tiene comportamiento inadecuado. Esta ambivalencia denota inmadurez, propia de su edad. El adolescente tiende a vivir un mundo ideal que le impide asumir el mundo real, lo que le hace endeble a ser influenciado por personas agresoras (pares). Pero mucho de lo que se vive es reflejado en el mundo mediático y hasta en la propia familia, con padres que discuten con frecuencia y dónde se impone el que más grita (gráfico 4).

Esta cotidianeidad de la violencia, desde dentro (familia) y desde fuera, con los programas televisivos, mal denominados de entretenimiento que refuerzan y pregonan abiertamente a la mujer como sexo débil y como objeto sexual, hacen que la violencia de género pase desapercibido para muchas de ellas. Un 10\% de adolescentes ya vivía situaciones que reproducen esta violencia (gráfico 4) y probablemente les parece normal porque viven en un cultura que privilegia la supremacía del varón. Bandura y Walters, citado por Salas, ${ }^{18}$ sostienen que los comportamientos antisociales, agresivos y violentos dependen esencialmente de un proceso de aprendizaje, siendo el componente principal la interacción con el ambiente.

En Manchay se vienen desarrollando proyectos orientados a disminuir y prevenir la violencia familiar desde hace algunos años, promoviendo una cultura de paz. Estos proyectos incluyen capacitación de los adolescentes de escuelas públicas y parroquiales, a fin de convertirlos en líderes activos en la comunidad; además de formar líderes sociales y educativos. Aunque el $50 \%$ de esa población ha recibido información sobre la prevención de la violencia familiar y sexual, éstas pueden pasar desapercibidas si se vive en un marco de situaciones conflictivas que desencadenan en violencia. ${ }^{19}$ Demás está decir que los medios de comunicación masiva, como la prensa escrita y televisiva, centran su información en noticias de porte violento, destacando los feminicidios que impactan y zozobran a la comunidad en general. 
Los resultados del estudio muestran la necesidad de seguir trabajando en el área de investigación, considerando que los y las adolescentes constituyen una población vulnerable, pero al mismo tiempo moldeable, es decir, que uniendo esfuerzos con los padres, líderes y autoridades se pueden crear nuevos modelos de desempeño personal y social, fundamentados en los derechos humanos de respeto e igualdad entre varones y mujeres.

Son los padres los primeros llamados a orientar a las jóvenes, además de discursos con ejemplos de vida, diálogo permanente y fortalecimiento de la autoestima, ayudándole a definir su carácter y personalidad. Proceso que debe ser fortalecido en la escuela, la comunidad mediante los grupos sociales con los que interacciona, como la red parroquial, la municipalidad, los centros de salud, entre otros.

Es imperativo desarrollar actividades a nivel local, que conlleven a la adopción de habilidades para la vida inmersa en la sana convivencia, las que ayuden al reforzamiento de una autoestima positiva, que permita valorarse, aceptarse y amarse; por otro lado, es necesario un trabajo multisectorial con las familias, a fin de fortalecer este vínculo que cada vez se deteriora en forma

\section{Correspondencia:}

Maria Inés Ruiz Garay

Correo electrónico: ines.ruiz@correo.eeplt.edu.pe

\section{REFERENCIAS BIBLIOGRÁFICAS}

1. Ruiz-Pérez I, Blanco-Prieto P, Vives-Cases C. Violencia contra la mujer en la pareja: determinantes y respuestas socio- sanitarias. Gac Sanit [serie de internet]. May 2004 [citado: 12 de ago 2014]; 18 (12): 4-12. Disponible en: http://scielo.isciii.es/scielo.php?pid=S021391112004000500003\&script=sci_arttext

2. Instituto Nacional de Estadística e Informática (INEI). Encuesta Demográfica y de Salud Familiar - ENDES. 2014 [internet]. Lima-Perú: INEI, abril 2015 [citado: 18 de may 2015]. p.490. Disponible en: http://www.inei.gob.pe/media/MenuRecursivo/ publicaciones_digitales/Est/Lib1211/pdf/Libro. pdf

3. Organización Mundial de la Salud [internet]. Ginebra: OMS; s.f. [actualizado: 2015; citado galopante; es el hogar donde se cristalizan los valores que llevarán a ese niño, futuro adolescente, a construir el mundo en el que todos merecen vivir, propiciando los cambios de conductas sociales negativos, rompiendo el circulo de violencia que se transmite de generación en generación.

Concluyendo, los resultados muestran presencia de factores de riesgos individuales y sociales, siendo necesario valorar las situaciones que circunscriben este proceso en el ámbito familiar y social, reconociendo que los padres y la relación que se establece con los mismos son relevantes en la identidad y amor propio de la adolescente. Especialmente porque las adolescentes son las futuras mujeres y madres del mañana, quienes se constituirán también en formadoras de nuevos seres humanos, ciudadanos responsables y respetuosos.

Dado la naturaleza del diseño del estudio, los resultados solo muestran la realidad de las adolescentes participantes, sin embargo puede ser reflejo de muchos grupos de adolescentes, lo que amerita que se continúe investigando en otras áreas y distritos del país, a fin de contar con una visión clara de la problemática en esta etapa previa que permitiría realizar un trabajo preventivo y de impacto para el presente y para el futuro.
12 de may 2015]. Género y salud de la mujer. Violencia contra la mujer infligida por su pareja; [aproximadamente 2 laudas]. Disponible en http://www.who.int/gender/violence/who_multi country_study/summary_report/chapter2/es/ind ex3.html

4. Tenorio CMF. Adolescencia, pareja y violencia familiar. Temát psicol [serie de internet]. 2011 [citado: 12 de ago 2014]; 7(1): 25-32. Disponible en: http://www.unife.edu.pe/publicaciones/revistas/ revista_tematica_psicologia_2011/tenorio_cast ro.pdf

5. Rey ACA. Prevalencia de factores de riesgo y problemáticas asociadas con la violencia en el noviazgo: una revisión de la literatura. Avances 
en Psicología Latinoamericana/Bogotá [internet]. 2008 [citado 15 sep 2014]; 26(2): 227-241. Disponible en: http://www.scielo.org.co/pdf/apl/v26n2/v26n2a 9.pdf

6. Alonso GE. Mujeres víctimas de violencia doméstica con trastorno de estrés postraumático: validación empírica de un programa de tratamiento [tesis doctoral]. Madrid-España: Universidad Complutense de Madrid; 2007[citado 12 de dic 2014]. Disponible en:

http://biblioteca.ucm.es/tesis/psi/ucmt29877.pdf

7. Nuñez AJ. La víctima [internet]. Sucre-Bolivia: Proyecto Sucre Ciudad Universitaria; 2004 [citado 10 de feb 2014]. [aproximadamente 05 laudas]. Disponible: http://www.nunezdearco.com/lavictima.htm

8. Corsi J. La violencia hacia la mujer en el contexto doméstico. México: Fundación Mujeres, 2012.

9. Nóblega MM, Muñoz VPV. Una aproximación cualitativa a la violencia hacia la mujer, en un asentamiento humano de Villa El Salvador. Liberabit [serie de internet]. Jul-dic 2009 [citado: 10 de ago 2013]; 15 (2): 95-108. Disponible en: http://www.scielo.org.pe/scielo.php?script=sci_ arttext\&pid=S1729-48272009000200004.

10.Gonzáles-Ortega I, Echeburúa PCE. Variables significativas en las relaciones violentas en parejas jóvenes: una revisión. Psicología conductual [internet]. 2008 [citado 20 abr 2012]; 16 (2): 207-225. Disponible en: http://www.ehu.eus/echeburua/pdfs/04GONZA LES.pdf

11. Moreno K, Cuevas JL. Violencia familiar y adicciones [internet]. Tlaxcala-México: Centro de Integración Juvenil, AC.; 2007 [citado 15 de ene 2015]. p.284. Disponible en: http://bibliotecadigital.tamaulipas.gob.mx/archi vos/descargas/d710cdc553885e58ba97257a3b9 56ea180196311.pdf

12.United Nations Childre's Fund. Adolescencia una etapa fundamental [internet]. New York: UNICEF; 2002 [citado 15 ene de 2015]. p.44. Disponible http://www.unicef.org/ecuador/pub_adolescenc e_sp.pdf

13.Garcia AGN. Antecedente de violencia doméstica y actitud violenta en hombres residentes en Manchayy. Lima [tesis de licenciatura]. Lima-Perú: Universidad Ricardo Palma; 2013.

14. Organización Panamericana de la Salud. Informe mundial sobre Violencia y Salud [internet]. Washington DC: OPS/OMS; 2002 [citado 15 de ago 2013]. p.62. Disponible en: http://www.who.int/violence_injury_preventio n/violence/world_report/en/summary_es.pdf

15.Malvaceda EE. Nakari: Formas culturales del sufrimiento. Universidad Nacional Mayor de San Marcos. Revista IIPSI [serie de internet]. 2010 [citado 15 de feb 2013]; 13 (2): 129-38. Disponible en: http://sisbib.unmsm.edu.pe/bvrevistas/investiga cion_psicologia/v13_n2/pdf/a08v13n2.pdf

16.La República. Cada día de 8 a 10 mujeres son maltratadas en Manchay [internet]. [Sociedad]. Lima-Perú: La República.pe; enero 4 del 2008 [citado 10 de abr 2013]; [aproximadamente 01 lauda]. Disponible en: http://larepublica.pe/0401-2008/cada-dia-de-8-10-mujeres-sonmaltratadas-en-manchay

17. Mora RC. Madres e hijas maltratadas: la trasmisión intergeneracional de la violencia doméstica en el Perú [internet]. Lima-Perú: Grade; 2013 [citado 15 de ene 2015].p.72. Disponible en: http://www.grade.org.pe/upload/publicaciones/ archivo/download/pubs/AI9.pdf

18. Salas BD, Baldeón SA. Criminalización de la violencia familiar desde una óptica crítica Lima-Perú: Universidad de Lima; 2009. p.223.

19.Escuela para el Desarrollo. Primer encuentro de proyectos e iniciativas relacionadas a la prevención y gestión de conflictos sociales [internet]. Lima-Perú: Escuela.org.pe; 2010 [citado 15 de ene 2015]; [aproximadamente 03 laudas]. Disponible en: http://www.escuela.org.pe/mos/prevencion-dela-violencia-domestica-en-manchay 